\title{
Using BIM to facilitate iterative design
}

\author{
M. H. Sakikhales \& S. Stravoravdis \\ Faculty of Architecture, Computing and Humanities, \\ Greenwich University, UK
}

\begin{abstract}
Architecture design practitioners typically generate and assess few design alternatives at the early stages of a project, before converging on a final design. Exploring design alternatives and understanding their impact on building energy performance leads to better performing building solutions. Therefore, any automatic process that gives the designer options to explore more alternatives and make decisions based on building performance would be of great benefit. If we look at the aerospace and automotive industries, they have developed multidisciplinary design optimization (MDO) methods, which are resulting in a significant reduction in the design cycle time and thus promoting more design iterations which then leads to improved product performance. MDO methods have been successfully applied in these industries, but their application to architecture practice has been comparatively modest. With the advent of BIM, however, it is now easier to facilitate the adoption of practices from other industries. This paper compares MDO processes in the Architecture, Aerospace and Automotive industries based upon data gathered on recent projects in each industry. It then reviews how iterative design and MDO process formalizes problem solving and coordination among groups working on the design of complex engineering systems. Finally, this paper investigates the feasibility of using BIM to facilitate an iterative design and MDO process which can result in the improvement in the number of design iterations of a building project.
\end{abstract}

Keywords: BIM, iterative design, early-stage design, multi-disciplinary optimization.

\section{Introduction}

Achieving sustainability targets for a building is highly depended on the early stage design phase when important decisions are made [1]. However, traditional 
architectural practices do not provide enough opportunities for analysis and further design exploration at the early stage design to achieve improved performance. In traditional design, 2D drawings were generated by architects first and then, a range of experts, including energy consultants participated and contributed to the project by generating their own analysis based on the architects' design. As the design evolved, these experts had to update their analysis with the ever changing design. Therefore, this process was time consuming and sometimes these experts could not respond to the design changes within the project timeline. Moreover, as this process is time, labour and cost intensive, it is typically done at the design development stage and the finalised design, which does not allow the design team to assess many of the design options generated at an earlier stage.

This lack of integration of relevant disciplines in the design process can lead to inefficient performance testing. However, other industries such as aerospace and automotive have developed multidisciplinary design optimization (MDO) methods, which are resulting in a significant reduction in the design cycle time. This, promotes more design iterations which then leads to improved product performance. MDO methods have been successfully applied in these industries, but their application to architecture practice has been comparatively modest. However, with the advent of BIM as a data rich, object-oriented, intelligent and parametric digital representation of a building in the AEC industry in recent years, it is now easier to facilitate the adoption of practices from other industries, because Building Information Modeling (BIM) allows for multi-disciplinary information to be overlaid in one model [1] and it creates the opportunity for sustainability analysis to be performed at the early stage design [2].

The main objective of this research is to investigate the feasibility of using BIM to facilitate an iterative design and MDO process which can result in the improvement in the number of design iterations of a building project. This paper compares design and optimization processes in the architecture, aerospace and automotive industries. It then reviews how iterative design and MDO can be adopted in the building industry and how BIM can facilitate them.

\section{The importance of design on building performance}

Building performance is the results of numerous factors such as building mass, building orientation, surface area-to-volume ratio, thermal insulation, natural lighting, natural ventilation, window areas and shading devices [3]. These aspects can greatly decrease building energy requirements and improve occupant comfort by collecting desirable forms of energy and protecting from undesirable ones [4]. For instance, in terms of using solar energy for heating, a well-designed building has the ability to collect solar energy through appropriate orientation, store energy in thermal mass and naturally distribute the stored solar energy back to the living space [5]. According to Jansson et al. [4] an optimized building orientation, shape, insulation and ventilation can reduce the heat consumption of a building up to $80 \%$. However, in order to achieve the highest level of performance, these aspects should be considered at the early stages of design [3]. It is also crucial to consider all possible options, as some of them potentially may contain better performing 
building solutions than anything previously considered. Conversely, architects and engineers often precede the design process with little or no information about the performance of the selected design alternative in comparison with other potential options.

\section{The importance of iterative design}

Iteration is defined as a process of repeating a set of steps until a desirable result is achieved [6] and any design process is by nature iterative [7]. In order to improve the specification of a product, it is crucial to generate as many design concepts as possible at the earliest stages [8] and to evaluate them and prepare feedback [9]. However, according to Pugh [8], there is the temptation to 'cut and run' and move towards final design development in engineering projects. Therefore, often, engineers and consultants instead of exploring and evaluating many alternatives, only analyse a chosen design option, which this could result in poor product performance.

\subsection{Design in engineering projects}

Ulrich and Eppinger [9] stated that the generic design development process in engineering projects consists of six phases as illustrated in Table 1. Other researchers, in general, also indicated the same phases in the design process, but with alternative name in some cases [10]. This process can be characterized as an iterative process, especially between phase 1 and phase 3 . This iterative process is crucial for generating value in design processes [10], because generating many alternatives increases the likelihood of identifying the best option [11]. Although the process of design refinement can be complicated and time-consuming [10], iteration results in detailed and well-tested specification which could proceed into further development [6]. It can therefore be argued that more building design iterations is a way towards better performing buildings.

Table 1: Generic development process, adopted from [9].

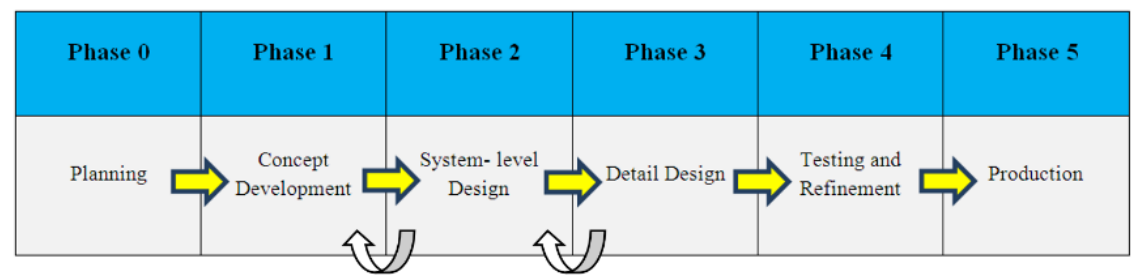

\subsection{Design in architecture and the building industry}

The RIBA Plan of Work is the most well-known model of building design and construction process. Table 2 shows a summary of this workflow. In this process, architects start the design process by defining their performance goal. Then, they generate some conceptual design alternative to meet these goals, but these 
alternatives are only considered in terms of aesthetics or finance and they rarely consider other criteria such as energy performance. Any performance analysis,typically starts after the developed design stage. This descriptive model is a point to point process, where each stage is separated from the previous one. Each stage starts when the previous one is completed and therefore cannot represent iterative processes [13]. As a result, any design iterations may identify as rework and may have the potential to increase the costs and time of the project [14].

Table 2: RIBA plan of work [12].

\begin{tabular}{|c|c|}
\hline \multicolumn{2}{|c|}{ RIBA Plan of Work } \\
\hline Stage 0 & Strategic Definition \\
\hline Stage 1 & Preparation and Brief \\
\hline Stage 2 & Concept Design \\
\hline Stage 3 & Developed Design \\
\hline Stage 4 & Technical Design \\
\hline Stage 5 & Construction \\
\hline Stage 6 & Handover and Close Out \\
\hline Stage 7 & In Use \\
\hline
\end{tabular}

Moreover, conventional project management techniques such as CPM and PERT which are very common in construction practices do not have the ability to represent feedback and iteration in the projects, mostly, because they only allow one-way progression in the activity process [13]. In an interview with 15 international building design practitioners, this process was found to be rigid and inflexible [14]. Implanting this stage to stage workflow increases the chance of discarding design alternatives at the early stage before their capacities are truly explored.

\section{Issues in using performance analysis at the early stage design}

There are several major challenges that need to be addressed in order to increase generating and analysing more design alternatives at the early stage design which are explained in the following sections.

\subsection{Interoperability}

One major challenge in integrating performance analysis at the early stage design is the interoperability between design tools and analysis tools [15]. Practitioners, 
instead of spending time on interpreting results and making decision, appear to be spending a significant amount of time on coordinating existing information. This coordinating process mostly consists of managing design information, including manual or semi-manual transcription and recoding of already existing information, manually integrating and representing this information and coordinating their solutions [16]. With the advent of BIM though, there is potential for a faster and more accurate data management, although many simulation tools currently only utilise BIM to import 3D geometry from a project.

\subsection{Lack of time}

Performance analysis is a time consuming process. An initial survey of 50 design professionals ( 5 architects, 45 multidisciplinary engineers) working at Ove Arup and Partners showed that architects and engineers spent over one month to generate and analyse a design alternative. So, in the conceptual design phase, they completed less than three iterations [16]. One reason that causes this issue is that architects and engineers spend $54 \%$ of their time on managing design information [16].

\subsection{Lack of feedback}

Decision making on design options needs a good communication to ensure that the needed information is available. The results of performance analysis have to feed back into design iteration fast enough to remain relevant for the current iteration [15]. However, many information exchanges can result to an increase in the duration of the design process, greater costs and wastage of resources which could affect the quality of the design [17]. Another problem is that after information is produced, little consideration is given as to how to represent them to facilitate multidisciplinary analysis [16].

These limitations prevent a more complete and systematic exploration of the design space based on multidisciplinary performance analysis. Other industries, such as aerospace have faced similar design challenges and in order to overcome these challenges, they have developed new approaches.

\section{Experiences from other industries}

Integration between vehicle components to achieve a certain level of performance is crucial in many industries such as aerospace and automotive. These industries consist of relatively large organizations with generally higher profits than the building design industry, which allows them to afford to invest in process engineering to develop processes and technologies that can achieve their requirements [13].

\subsection{Aerospace industry}

In 1998, Boeing began a project to design a hypersonic vehicle with a mission to deliver a payload into the upper stratosphere. Integration between the different 
components and the external geometry was crucial to achieve the expected performance level. However, after six years of work with a similar workflow in the building industry, the design team could not prepare a design that could meet all requirements. Therefore, in 2002, Boeing adopted a new methodology to support multidisciplinary analysis leading to improved product performance [16].

This process had three steps: (1) the design team produces a parametric vehicle topology and selects the range of parameters to be varied. (2) Each discipline analyses this model and produces their results. (3) A Design Explorer controls parameter configurations using statistical methods to explore the entire design space. Finally, an optimizer uses the performance feedback obtained to find the best design option [16]. Using this method resulted in a significant increase in the number of design iterations, as shown in Table 3, where the new method is compared to the legacy design method in a given twelve weeks period. With the previous method, the design team analysed 116 engine inlet flow paths and 12 different vehicle configurations over the course of eight years, while using the MDO process, the design team successfully analysed 3900 engine inlet flow paths and 98 different vehicle configurations in six days [15]. This dramatic change resulted in the significantly improved vehicle configuration.

Table 3: Comparison of traditional and MDO process for the design of a hypersonic vehicle [16].

\begin{tabular}{|c|c|c|c|c|c|c|c|c|}
\hline \multirow{2}{*}{ Design method } & \multirow{2}{*}{\multicolumn{5}{|c|}{ Time }} & \multicolumn{2}{|c|}{ Iteration Duration } & \multirow{2}{*}{$\begin{array}{l}\text { Number } \\
\text { of } \\
\text { iterations }\end{array}$} \\
\hline & & & & & & Primary & Later & \\
\hline $\begin{array}{c}\text { Previous } \\
\text { method }\end{array}$ & $8 \%$ & $32 \%$ & & $50 \%$ & $10 \%$ & 6 weeks & 4 weeks & 2.5 \\
\hline MDO & $26 \%$ & & $18 \%$ & $8 \%$ & $48 \%$ & 14 weeks & $1.5 \mathrm{Hrs}$ & $>1000$ \\
\hline
\end{tabular}

Specification

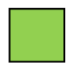

Execution Management Reasoning

\subsection{Automotive industry}

In a recent research project, Mazda Motor Corporation in Japan developed a new MDO process for structural analysis of the Mazda CX-5 vehicle. During this process, a variety of computer-aided engineering (CAE) software such as Abaqus, LS-DYNA and Nastran were used for modelling. Then, a complex analysis system was produced to optimize vehicle body behaviour in each of the pre-defined targets and then to identify the final design alternative that can achieve all these 
targets together at the lightest possible weight. Next, a variety of Design of Experiments (DOE) methods and approximation models were employed and then, a comparison between the different behaviours was conducted manually which took a great deal of time. After that, the design team decided to turn to the process automation design exploration. Using this process and setting up the MDO within an automated workflow, resulted in significant reduction in the analysis setup and runtime. As a result, a 3.4\% reduction in weight over the previous design of the CX-5 was achieved which was a great achievement due to its significant effects on fuel consumption. By including an MDO step within the structure development process, more options can be analysed in a shorter period of time. In order to complete a MDO, a higher computational resource needs to be allocated, but the potential mass savings justify the effort [19].

There are fundamental differences between these industries and architectural practice, thus limiting the adoption of practices from one to the other. However, with the advancement in new technologies in the building industry such as 3D printing and robotics, a lot of which have been used already by the aerospace and automotive industries, there is a potential to adopt more methods and practices from other industries. For instance, the integration of BIM and new performance analysis methods could allow architects and engineers to overcome a lot of these limitations and to more rapidly generate design iterations and analyse them at the early stage design.

\section{How BIM can facilitate iterative design}

Although there is a lot of research on BIM technology and using BIM in collaborative design, there is comparatively less research on the integration of BIM and building performance [20]. However, BIM has the potential to facilitate energy performance analysis and has introduced solutions to overcome current barriers. Moreover, BIM allows other design processes such as parametric modelling to integrate in the design process which can facilitate the iterative design process.

\subsection{Overcoming barriers}

Various methods have been proposed to overcome data interoperability obstacles between different disciplines or software. (1) A closed system of software which are compatible to each other and unified through an exclusive data format. (2) A loose system of different tools and software which can share their data through a standardised data format [15]. These approaches can also be named as Closed BIM and Open BIM. File formats such as the IFC and gbXML have been developed to facilitate data exchange processes without any need to remodel the same project [20] which could result in significant time savings.

In addition, some BIM platforms have their own compatible performance analysis tools such as GBS for Revit, EcoDesigner for ArchiCAD and AECOsim for Bentley BIM, which improves on the data exchange problem. Other analysis software have also developed plug-ins for BIM platforms. For instance, IES has a plug in for Revit which can facilitate data exchange between the two software. 
Another example is Sefaira, which can run directly within Revit and provides analysis with no need to exchange file format.

Feedback from performance analysis produces new information which is critical for the design teams as it could lead them to refine the design before proceeding [9]. This feedback process needs an effective collaboration and communication and BIM can play a major role in this process. One way to accelerate feedback of analysis results is using cloud based platforms. Using cloud based communication platforms is important due to the geographic distribution of construction project teams [15]. There are many online collaboration platforms (OCP) that can facilitate collaboration and communication between various disciplines.

\subsection{BIM and iterative design}

With the advent of parametric modelling, working in a linear fashion is no longer necessary because the designers can modify the model at any time during the design process and the results would be updated automatically. Moreover, current parametric design tools such as Grasshopper provide the ability to rapidly generate design iterations through the use of parameters and algorithms. Although research on the potential of integrating BIM, building performance optimization and parametric modelling is still ongoing, some research has already been carried out.

Welle et al. [21] have developed a thermal optimization methodology called "Thermal Opt" which enables a larger number of design iterations to be analysed at the early stage design. Rahmani Asl et al. [22] have created a prototypical system interface between BIM and energy simulation tools which can help architects to develop BIM-based parametric models and simulation. Lin and Gerbe have used BIM models and generative algorithms to develop a MDO framework to use energy performance as feedback for architects at the early stage design [22]. In addition, commercial developers have also developed and introduced new plug in software for BIM platforms such as Dynamo and Generative Components (GC) that enable researchers to expand their investigation. Rahmani Asl et al. [23] developed a prototype using Dynamo, Revit and GBS to run parametric simulations for a sample project. A multi objective optimization algorithm is used to optimize multiple conflicting objectives and approach to a set of optimal solutions and the system was run for 1000 times, which took around 3 hours overall to achieve the optimal design option [23]. Mueller [24] developed a prototype with the set of Bentley software including GC, STAAD and DARWIN. The system used the Energy Plus engine for energy analysis and computed the various simulations and generated results which then transferred to the DARWIN optimization framework which includes a prototype implementation of a multiobjective genetic algorithm (MOGA) for further processing and finding of the optimal alternative [24].

\section{Discussion and conclusion}

The integration of BIM and parametric modelling can considerably advance the optimization of building energy [22] and can help designers to generate a large 
number of design solutions. Performance analysis can be automated with the help of generative algorithms and MDO methods. Taking to account aerospace and automotive industries' experience and recent architecture and building research, it can be concluded that this system needs four important elements: (1) Parametric geometry modelling, (2) Integrated platform, (3) Multi-disciplinary optimizer and (4) Genetic algorithm. The relationship between these elements is showed in Figure 1. It is important to have a parametric model from the beginning, as it enables the genetic algorithm to explore design alternatives, while facilitating feedback and results to instantly show on the model. An integrated platform helps to evaluate many design options in a short time. Finally and optimizer or decision making system is required in order to quantify the system in terms of objectives and constraints. It is important to identify parameters and constraints and understand how they behave in the model and how they can impact on the project's objective. However, for better interoperability and less coding errors, it is better and simpler to use compatible software. This could be a group of software from the same developer or a group of software that already have been using a compatible code.

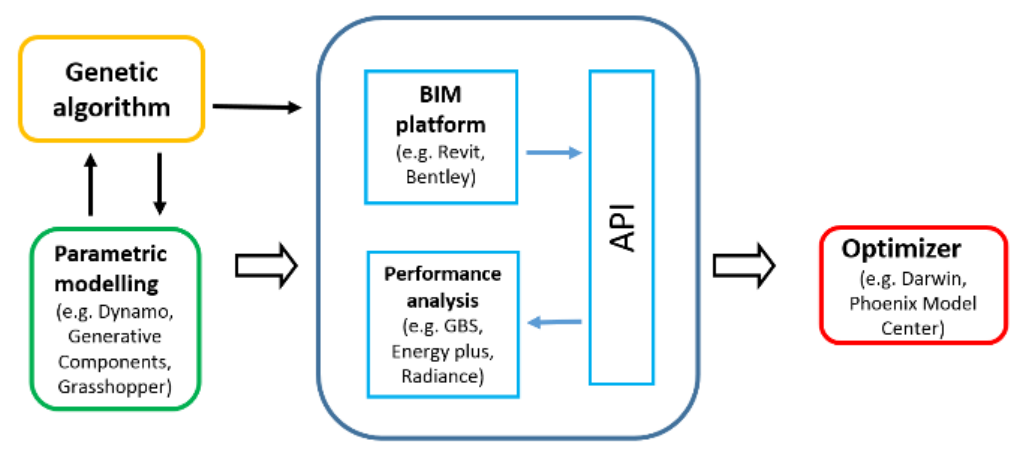

Figure 1: Relationship between platforms in BIM base iterative design.

Nevertheless, establishing this system in the building industry is not easy, as other industries have passed the trial and error phase and their practitioners have become familiar with this system and its required skills. Although many architects are familiar with these skills, they are still complicated and sophisticated for practitioners and a lot of time and training is needed to become a part of usual architectural modelling practice. Moreover, the building industry does not have the ability to produce and analyse the prototype and feed the information back to the detailed design stage. Therefore, even with all the new technologies it is difficult to reach the level of performance that other industries can achieve. In addition, the length of the design process is another important difference in these industries. The average design time in the aerospace industry is 3-4.5 years and in automotive industry is around 3.5 years [9], while a typical family house could be designed, built and introduced to the market in less than a year. So, most of the construction firms cannot invest a great deal of time on the design phase and their projects have to be designed in a few months. Moreover, almost all building 
projects in the UK have to gain planning permission from their local authorities. Therefore, it is preferred not to spend so much at the design phase without having secured planning consent.

Another major challenge of adopting this process is to define the parameters. Other industries already have spent a lot of time and money to understand relevant parameters and how to control them in a multi-disciplinary process. For an organisation in the building industry, it will take a significant amount of time to determine suitable parameters and constraints and identify their behaviour to produce suitable design alternatives.

In order to adopt this new approach, the role of architects and engineers needs to change. Architects are no longer just designers who can generate great ideas. They have to know more and more about the role of sustainable design factors and they have to think, design and program in a parametric way. On the other hand, engineers have to change their role from someone who can manipulate data and perform calculations, to someone who can identify important parameters in different disciplines and determine rules between them.

\section{Future work}

This work is a part of an ongoing $\mathrm{PhD}$ research project on the integration of BIM and building performance. Research is ongoing in order to gain a clearer insight on using MDO at the early stage design and how BIM can facilitate this process.

\section{References}

[1] Azhar, S. Brown, J. \& Farooqui, R., BIM-based Sustainability Analysis: An Evaluation of Building Performance Analysis Software, Proc. of the 45th Associated Schools of Construction International Conference, Gainesville, 2009.

[2] Schlueter, A. \& Thesseling, F., Building information model based energy/exergy performance assessment in early design stages. Automation in Construction, vol. 18, pp. 153-163, 2009.

[3] Smeds, J \& Wall, M., Enhanced energy conservation in houses through high performance design. Energy and Buildings, vol. 39, pp. 273-278, 2007.

[4] Jansson, G. Schade, J. \& Olofsson, T., Requirements management for the design of energy efficient building. Journal of Information Technology in Construction, vol. 18, pp. 321-337, 2013.

[5] Fosdick, J. \& T. C. Homes, Passive Solar Heating, Online. www.wbdg.org/resources/psheating.php

[6] Butler, J. Holden, K. \& Lidwell, W. Universal Principles of Design, Revised and Updated, Beverly: Rockport Publishers, 2010.

[7] Ballard, G., Positive vs negative iteration in design, Proc. of the 8th Conference of the International Group for Lean Construction, Brighton, 2000.

[8] Pugh, S. Total Design Integrated Methods for Successful Product Engineering, Essex: Pearson Education Limited, 1991. 
[9] Ulrich, K.T. \& Eppinger, D. S., Product Design and Development, Fifth ed., New York: McGraw Hill, 2012.

[10] Cross, N. Engineering Design Methods Strategies for Product Design, Fourth ed., Sussex: John Willey \& Sons, 2011.

[11] Stoll, H. W., Product Design Methods and Practices, New York: Marcel Dekker, 1999.

[12] RIBA, www.ribaplanofwork.com

[13] Pektasx, S. T. \& Pultar, M., Modelling detailed information flows in building design with the parameter based design structure matrix, Design Studies, pp. 99-122, 2006.

[14] Hopfe, C. J., Struck, C., Hensen, J. \& Böhms, M., Adapting advanced engineering design approaches to building design-potential benefits, Proc. of the 6th postgraduate research Conference in the built and human environment, Manchester, 2006.

[15] Mueller, V., Crawley, D. \& Deb, P., Second iteration of cloud-based analysis and optimization framework, Proc. of the 13th Conference of International Building Performance Simulation Association, Chambéry, 2013.

[16] Flager, F. and Haymaker, J., A Comparison of Multidisciplinary Design, Analysis and Optimization Processes in the Building Construction and Aerospace, Standford University, 2009.

[17] Mujumdar, P. \& Maheswari, J. U., A design iteration framework for construction project, Proc. of the RICS Cobra, New Delhi, 2013.

[18] 3ds, www.3ds.com

[19] Sheldon, A., Helwig, E. \& Cho, Y.-B., Investigation and Application of Multi-Disciplinary Optimization for Automotive Body-in-White Development, Proc. of the 8th European LS-DYNA Users Conference, Strasbourg, 2011.

[20] Zanni, M., Soetanto, R. \& Ruikar, K., Facilitating BIM-based sustainability analysis and communication in building design process, Proc. of the 6th Civil Engineering Conference in Asia Region, Jakarta, 2013.

[21] Welle, B., Haymaker, J. \& Rogers, Z., ThermalOpt: A Methodology for Automated BIM-Based Multidisciplinary Thermal Simulation for Use in Optimization Environments, Stanford University, 2011.

[22] Rahmani Asl, M., Zarrinmehr, S. \& Yan, W., Towards BIM-based Parametric Building Energy Performance Optimization, Proc. of the 33rd Annual Conference of the Association for Computer Aided Design in Architecture, Cambridge, 2013.

[23] Rahmani Asl, M., Bergin M., Menter, A. \& W. Yan, BIM-based Parametric Building Energy Performance Multi-Objective Optimization, Proc. of the 32nd International Conference on Education and research in Computer Aided Architectural Design in Europe, Newcastle, 2014.

[24] Mueller, V., Second generation prototype of a design performance optimization framework, Proc. of the 7th International Conference of the Arab Society for Computer Aided Architectural Design, Jaddah, 2014. 